University of Wollongong

Research Online

Faculty of Informatics - Papers (Archive)

Faculty of Engineering and Information

Sciences

January 2003

\title{
Frame analysis for biorthogonal cosine-modulated filterbanks
}

Alfred Mertins

University of Wollongong, mertins@uow.edu.au

Follow this and additional works at: https://ro.uow.edu.au/infopapers

Part of the Physical Sciences and Mathematics Commons

\section{Recommended Citation}

Mertins, Alfred: Frame analysis for biorthogonal cosine-modulated filterbanks 2003.

https://ro.uow.edu.au/infopapers/92

Research Online is the open access institutional repository for the University of Wollongong. For further information contact the UOW Library: research-pubs@uow.edu.au 


\title{
Frame analysis for biorthogonal cosine-modulated filterbanks
}

\begin{abstract}
This paper addresses the efficient computation of frame bounds for cosine-modulated filterbanks. We derive explicit expressions for the eigenvalues of the frame operator that can be easily computed from the prototype's polyphase components. The number of channels and the downsampling factor may be even or odd, and the oversampling factor is supposed to be an integer. The analysis of low-delay, biorthogonal filterbanks; shows that prototypes solely designed to minimize the stopband energy may lead to wide open frames and, thus, to an undesirable numerical behavior. Because the computational cost of determining the frame bounds with the proposed method is very low, we can directly use the bounds during prototype optimization and obtain prototypes with minimum stopband energy under the condition of fixed frame bounds. Various design examples are presented.
\end{abstract}

\section{Disciplines}

Physical Sciences and Mathematics

\section{Publication Details}

This article was published as: Mertins, A, Frame analysis for biorthogonal cosine-modulated filterbanks, IEEE Transactions on Signal Processing, January 2003, 51(1), 172-181. Copyright IEEE 2003. 


\title{
Frame Analysis for Biorthogonal Cosine-Modulated Filterbanks
}

\author{
Alfred Mertins, Member, IEEE
}

\begin{abstract}
This paper addresses the efficient computation of frame bounds for cosine-modulated filterbanks. We derive explicit expressions for the eigenvalues of the frame operator that can be easily computed from the prototype's polyphase components. The number of channels and the downsampling factor may be even or odd, and the oversampling factor is supposed to be an integer. The analysis of low-delay, biorthogonal filterbanks shows that prototypes solely designed to minimize the stopband energy may lead to wide open frames and, thus, to an undesirable numerical behavior. Because the computational cost of determining the frame bounds with the proposed method is very low, we can directly use the bounds during prototype optimization and obtain prototypes with minimum stopband energy under the condition of fixed frame bounds. Various design examples are presented.
\end{abstract}

Index Terms-Biorthogonal filterbanks, cosine-modulated filterbanks, frame analysis, low delay.

\section{INTRODUCTION}

C OSINE-MODULATED filterbanks are very popular in signal processing because of their low design and implementation costs. The first designs of perfect reconstruction (PR) cosine-modulated filterbanks were carried out to yield paraunitary filterbanks [1]-[4]. Paraunitary filterbanks use linear-phase prototypes, and the delay of the entire analysis/synthesis system is determined by the length of the prototype. More recently, biorthogonal, low-delay filterbanks have been introduced [5]-[11]. Such designs are attractive because they allow the choice of the overall system delay independently of the lengths of the filters involved. A collection of general PR conditions for critical sampling can be found in [10]. Design methods for finite impulse response (FIR) oversampled filterbanks were considered in [9] and [12]. Infinite impulse response (IIR) filterbanks were designed in [11].

The quality criterion commonly used in the design of both paraunitary and biorthogonal filterbanks is the minimization of the stopband energy of the prototype [8]-[11]. Design examples have shown that for a fixed delay the prototypes for biorthogonal filterbanks can have much higher stopband attenuation than the ones for paraunitary filterbanks. However, examples also show that for biorthogonal filterbanks, the good properties of a prototype do not necessarily translate into good properties of the modulated filters. In this paper, we will study this phenomenon using frame analysis.

Manuscript received September 14, 2001; revised August 7, 2002. The associate editor coordinating the review of this paper and approving it for publication was Dr. Helmut Boelcskei.

The author is with the School of Electrical, Computer, and Telecommunication Engineering, University of Wollongong, Wollongong, Australia (e-mail: mertins@uow.edu.au).

Digital Object Identifier 10.1109/TSP.2002.806572
Frame analysis is a powerful tool to assess the properties of filterbanks and transforms [12]-[16]. Frames for general, oversampled filterbanks have been studied in [14] and [15]. The special case of cosine-modulated filterbanks has been addressed in [12], where the aim was mainly to design filterbanks with tight frames (i.e., filterbanks that provide unitary transforms). In [16], frame analysis was used to assess the properties of biorthogonal two-channel filterbanks for image compression purposes where the energy of quantization noise is to be estimated in the subband domain.

In this paper, we use frame analysis to assess and design biorthogonal, cosine-modulated filterbanks. Based on the general methods proposed in [14] for determining frame bounds through an eigenanalysis of a frequency-dependent matrix, which is known as the frame operator, we derive new expressions for the frame bounds that are very easy and efficient to compute. In particular, we will derive explicit expressions for the eigenvalues of the frame operator. For the special case of critically sampled filterbanks, we will present an even more efficient method that finds the bounds directly from Fourier transforms of the autocorrelation sequences of the prototype's polyphase components. Because the computational cost of determining the frame bounds is very low, we can directly use the bounds during prototype optimization and obtain prototypes with minimum stopband energy under the condition of fixed frame bounds.

\section{A. Notation}

Matrices and vectors are printed in boldface. $I_{M}$ and $\boldsymbol{J}_{M}$ denote the $M \times M$ identity and counter identity matrices, respectively. The term diag $[\cdot]$ denotes the formation of a diagonal matrix. $\|\cdot\|$ means the Euclidean norm of a vector. $\mathbb{Z}$ is the set of all integers, $\mathbb{R}$ is the set of all real, and $\mathbb{C}$ is the set of all complex numbers. $[\cdot]$ and $|\cdot|$ mean rounding to the next larger/smaller integer, respectively. The asterisk $*$ denotes convolution.

\section{COSINE-Modulated FiLTERBANKS}

In this paper, we consider both critically sampled and oversampled cosine-modulated filterbanks. Fig. 1 shows the filterbank in direct form, where $h_{k}(n)$ and $g_{k}(n), k=0, \ldots, M-1$ denote the impulse responses of the analysis and synthesis filters, respectively. $M$ is the number of channels, and $N$ is the ratio between the sampling rate at the input and in the subbands. $M$ may be even or odd, but the oversampling factor $L=M / N$ is restricted to be an integer. The following type of modulation is used to derive the analysis and synthesis filters from proto- 


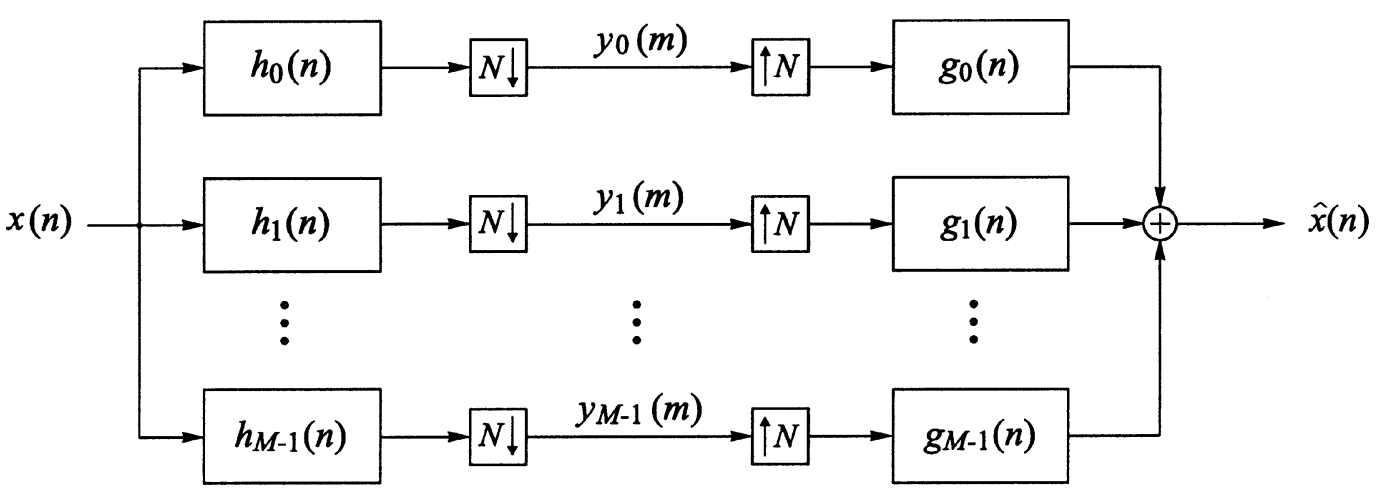

Fig. 1. $M$-band analysis and synthesis filterbanks with sampling rate ratio $N$.

types with impulse responses $p(n)$ and $q(n)$

$$
\begin{aligned}
& h_{k}(n)=\frac{1}{\sqrt{L}} p(n) c_{1, k}(n) \\
& g_{k}(n)=\frac{1}{\sqrt{L}} q(n) c_{2, k}(n)
\end{aligned}
$$

where

$$
\begin{aligned}
c_{1, k}(n)= & \sqrt{\frac{2}{M}} \\
& \times \cos \left[\frac{\pi}{M}\left(k+\frac{1}{2}\right)\left(n-\frac{D}{2}\right)+(-1)^{k} \frac{\pi}{4}\right] \\
c_{2, k}(n)= & \sqrt{\frac{2}{M}} \\
& \times \cos \left[\frac{\pi}{M}\left(k+\frac{1}{2}\right)\left(n-\frac{D}{2}\right)-(-1)^{k} \frac{\pi}{4}\right] .
\end{aligned}
$$

$D$ is the overall delay of the analysis/synthesis system and is assumed to be of the form

$$
D=2 s M+2 M-1
$$

where $s$ is an integer. Note that this is the most commonly used delay because it does not put severe restrictions on the prototype's coefficients. For example, it automatically occurs in the case of paraunitary cosine-modulated filterbanks. For other delays, some of the prototype's polyphase components have to degrade to simple delays or even zero sequences; see [10]. The range for $n$ in (1) depends on the filter lengths, which are, in general, independent of the delay $D$.

For further analysis, it is convenient to describe the filterbank via its analysis and synthesis polyphase matrices $\boldsymbol{E}^{(L)}(z)$ and $\boldsymbol{R}^{(L)}(z)$. The superscript $(L)$ denotes the oversampling factor. Given the filters in (1) with the modulation according to (2), the matrices $\boldsymbol{E}^{(L)}(z)$ and $\boldsymbol{R}^{(L)}(z)$ can be expressed as [9]

$$
\boldsymbol{E}^{(L)}(z)=\frac{1}{\sqrt{L}} \boldsymbol{C}_{1}\left[\begin{array}{c}
\boldsymbol{P}_{0}\left(z^{2 L}\right) \\
z^{-1} \boldsymbol{P}_{1}\left(z^{2 L}\right) \\
\vdots \\
z^{-(2 L-1)} \boldsymbol{P}_{2 L-1}\left(z^{2 L}\right)
\end{array}\right]
$$

$$
\begin{aligned}
\boldsymbol{R}^{(L)}(z)= & \frac{1}{\sqrt{L}}\left[z^{-(2 L-1)} \boldsymbol{J}_{N} Q_{2 L-1}\left(z^{2 L}\right) \boldsymbol{J}_{N},\right. \\
& z^{-(2 L-2)} \boldsymbol{J}_{N} Q_{2 L-2}\left(z^{2 L}\right) \boldsymbol{J}_{N}, \ldots \\
& \left.\ldots, \boldsymbol{J}_{N} \boldsymbol{Q}_{0}\left(z^{2 L}\right) \boldsymbol{J}_{N}\right] \boldsymbol{C}_{2}^{T} .
\end{aligned}
$$

In (4) and (5), $\boldsymbol{C}_{1}$ and $\boldsymbol{C}_{2}$ are $M \times 2 M$ cosine modulation matrices given by

$$
\left[\boldsymbol{C}_{1}\right]_{k, n}=c_{1, k}(n), \quad\left[\boldsymbol{C}_{2}\right]_{k, 2 M-1-n}=c_{2, k}(n)
$$

with $k=0,1, \ldots, M-1$ and $n=0,1, \ldots, 2 M-1$. The matrices $\boldsymbol{P}_{k}\left(z^{2 L}\right)$ are defined as (7), shown at the bottom of the page, where $P_{j}(z), j=0,1, \ldots, 2 M-1$ are the type- 1 polyphase component of the analysis prototype given by

$$
P_{j}(z)=\sum_{\ell} p_{j}(\ell) z^{-\ell}, \quad p_{j}(\ell)=p(2 \ell M+j) .
$$

The matrices $\boldsymbol{Q}_{k}(z)$ have the same definition as $\boldsymbol{P}_{k}(z)$, only with $P_{k}(z)$ replaced by the type-1 polyphase components $Q_{k}(z)$ of the synthesis prototype. The matrices $\boldsymbol{C}_{1}$ and $\boldsymbol{C}_{2}$ have the properties

$$
\boldsymbol{C}_{2}^{T} \boldsymbol{C}_{1}=\left[\begin{array}{cc}
(-1)^{s} \boldsymbol{I}_{M}+\boldsymbol{J}_{M} & \mathbf{0} \\
\mathbf{0} & (-1)^{s} \boldsymbol{I}_{M}-\boldsymbol{J}_{M}
\end{array}\right]
$$

and

$$
\boldsymbol{C}_{1}^{T} \boldsymbol{C}_{1}=\boldsymbol{C}_{2}^{T} \boldsymbol{C}_{2}=\left[\begin{array}{cc}
\boldsymbol{I}_{M}+(-1)^{s} \boldsymbol{J}_{M} & \mathbf{0} \\
\mathbf{0} & \boldsymbol{I}_{M}-(-1)^{s} \boldsymbol{J}_{M}
\end{array}\right] .
$$

With the polyphase matrices $\boldsymbol{R}^{(L)}(z)$ and $\boldsymbol{E}^{(L)}(z)$, the perfect reconstruction conditions can be formulated as

$$
\boldsymbol{R}^{(L)}(z) \boldsymbol{E}^{(L)}(z)=z^{-2 s L-2 L-1} \boldsymbol{I}_{N} .
$$

Expanding (11) using (4), (5), and (9) and replacing $-z^{2 L}$ by $z$ in the expression obtained yields the following PR conditions on the prototype [9]:

$$
\begin{aligned}
\sum_{\ell=0}^{2 L-1} P_{k+\ell N}(z) Q_{2 M-1-k-\ell N}(z) & =L z^{-s} \\
k & =0, \ldots,\left\lceil\frac{N}{2}\right\rceil-1 .
\end{aligned}
$$

$$
\begin{gathered}
\boldsymbol{P}_{k}\left(z^{2 L}\right)=\operatorname{diag}\left[P_{k N}\left(-z^{2 L}\right), P_{k N+1}\left(-z^{2 L}\right), \ldots, P_{k N+N-1}\left(-z^{2 L}\right)\right] \\
k=0,1, \ldots, 2 L-1
\end{gathered}
$$


Note that (12) shows the PR conditions on the prototype for $L$ times oversampled, biorthogonal, cosine-modulated filterbanks with the delay in (3). In the special case of paraunitary filterbanks, a linear-phase (symmetric) prototype is required, and the same prototype is to be used for analysis and synthesis. Equation (12) then leads to the following conditions on paraunitary prototypes [9]:

$$
\begin{aligned}
& \sum_{\ell=0}^{2 L-1} P_{k+\ell N}(z) P_{k+\ell N}\left(z^{-1}\right)=L \\
& \quad k=0, \ldots,\left\lceil\frac{N}{2}\right\rceil-1 .
\end{aligned}
$$

\section{Frame ANALYSIS}

\section{A. Definition and Meaning of Frames}

Let $h_{k, m}, k=0,1, \ldots, M-1, m \in \mathbb{Z}$ be vectors containing the filter coefficients $h_{k}(m M-n)$, and let $\boldsymbol{x}$ be a vector containing the input samples $x(n)$. Assuming that $\boldsymbol{x} \in \ell^{2}(\mathbb{Z})$, the following inequality can be stated:

$$
A\|\boldsymbol{x}\|^{2} \leq \sum_{k=0}^{M-1} \sum_{m=-\infty}^{\infty}\left|\left\langle\boldsymbol{x}, \boldsymbol{h}_{k, m}\right\rangle\right|^{2} \leq B\|\boldsymbol{x}\|^{2} \forall \boldsymbol{x} \in \ell^{2}(\mathbb{Z})
$$

with some $A, B \in \mathbb{R}$. If $A>0$ and $B<\infty$, the values $A$ and $B$ are called frame bounds, and the set $\boldsymbol{h}_{k, m}$ is called a frame for $\ell^{2}(\mathbb{Z})$. The frame bounds themselves can be seen as indicators of the numerical properties of the filterbank. If the set $\boldsymbol{h}_{k, m}$ constitutes a frame, then there exists a corresponding PR synthesis filterbank with filters $\boldsymbol{g}_{k, m}$ that also constitutes a frame with frame bounds $A^{\prime}$ and $B^{\prime}$ such that

$$
A^{\prime}\|x\|^{2} \leq \sum_{k=0}^{M-1} \sum_{m=-\infty}^{\infty}\left|\left\langle\boldsymbol{x}, \boldsymbol{g}_{k, m}\right\rangle\right|^{2} \leq B^{\prime}\|\boldsymbol{x}\|^{2} \forall \boldsymbol{x} \in \ell^{2}(\mathbb{Z}) .
$$

Under certain conditions, the bounds are related as $A^{\prime}=1 / B$ and $B^{\prime}=1 / A$. We will return to this point at the end of Section III-B2.

In the special case that $A=B$, the frame is called a tight frame. If $A=B=1$, the filterbank is paraunitary, and (14) reduces to $\sum_{k=0}^{M-1} \sum_{m=-\infty}^{\infty}\left|\left\langle\boldsymbol{x}, \boldsymbol{h}_{k, m}\right\rangle\right|^{2}=\|\boldsymbol{x}\|^{2}$. If $A=B=1$ and the filterbank is critically sampled, then the vectors $\boldsymbol{h}_{k, m}$ form an orthonormal basis for $\ell^{2}(\mathbb{Z})$, and the above-mentioned formula for energy preservation becomes Parseval's identity. Further discussion of the effects of the frame bounds on the filterbank properties (including the case where $A \approx B$ ) can be found in [14]. ${ }^{1}$

In general, the smaller the ratios $B / A$ and $B^{\prime} / A^{\prime}$, the better the numerical properties of the filterbank will be. If $B / A$ and $B^{\prime} / A^{\prime}$ are close to one, then the filterbank can be regarded as being almost paraunitary, and the assumption of energy preservation may be used without much error when relating the energy of the subband signals to the energy of the input or output signal of a filterbank.

\footnotetext{
${ }^{1}$ Note that in [14], the filters were normalized to have unit energy so that tight frames were obtained with $A=B=M / N$.
}

Having almost paraunitary filterbanks is particularly useful in source coding where operational rate-distortion algorithms are to be applied in the subband domain, and exact paraunitaryness cannot be achieved because of other requirements such as linear phase or low delay. For almost paraunitary filterbanks, the squared reconstruction error at the filterbank output is close to the squared quantization error in the subband domain. Thus, a bit allocation optimized for minimum error in the subbands will then be near-optimal for the final output signal. With frame analysis, it is possible to exactly determine the maximum deviation between the errors in the subband domain and at the filterbank output. With $\varepsilon(n)$ denoting the output error and $q_{k}(n)$ denoting the quantization error in band $k$ at time index $n$, the following inequality holds [16]:

$$
\begin{aligned}
A^{\prime} \sum_{k=0}^{M-1} \sum_{n=-\infty}^{\infty}\left|q_{k}(n)\right|^{2} & \leq \sum_{n=-\infty}^{\infty}|\varepsilon(n)|^{2} \\
& \leq B^{\prime} \sum_{k=0}^{M-1} \sum_{n=-\infty}^{\infty}\left|q_{k}(n)\right|^{2} .
\end{aligned}
$$

In (16), it is assumed that the entire error energy is finite, which is typically the case for real-world, time-limited signals.

\section{B. Computation of Frame Bounds for Cosine-Modulated Filterbanks}

Algorithms for the computation of the frame bounds have been described in [14] and [16]. We follow the method in [14] because it can be most easily applied to cosine-modulated filterbanks. We will first look at the analysis filterbank and derive explicit expressions for the eigenvalues of the frame operator. In a second step, we will consider the frame bounds for the synthesis filterbank and relate them to those of the analysis bank. Then, we will derive a simplified method for computing the frame bounds in the critically sampled case.

1) Frame Bounds for the Analysis Filterbank: Let

$$
\boldsymbol{S}(z)=\tilde{\boldsymbol{E}}^{(L)}(z) \boldsymbol{E}^{(L)}(z)
$$

where $\tilde{E}^{(L)}(z)$ is the paraconjugate of the analysis polyphase matrix $\boldsymbol{E}^{(L)}(z)$, that is, $\tilde{\boldsymbol{E}}^{(L)}(z)=\left[\boldsymbol{E}^{(L)}(z)\right]^{H}$ for $|z|=1$. Because cosine-modulated filterbanks have real-valued coefficients, we may write $\tilde{\boldsymbol{E}}^{(L)}(z)=\left[\boldsymbol{E}^{(L)}\left(z^{-1}\right)\right]^{T}$. Now, let us denote the eigenvalues of $\boldsymbol{S}\left(e^{j \omega}\right)$ as $\lambda_{k}(\omega), k=0,1, \ldots, N-1$. The frame bounds $A$ and $B$ are given by [12], [14]

$$
\begin{aligned}
& A=\operatorname{liss}_{\omega \in[0,2 \pi), k=0,1, \ldots, N-1} \lambda_{k}(\omega) \\
& B=\underset{\omega \in[0,2 \pi), k=0,1, \ldots, N-1}{\operatorname{ess} \sup _{k}} \lambda_{k}(\omega)
\end{aligned}
$$

where the symbols "ess inf" and "ess sup" denote the essential infimum and supremum, respectively. The matrix $\boldsymbol{S}\left(e^{j \omega}\right)$ represents the frame operator for the analysis filterbank.

In the following, we derive explicit expressions for the frame bounds, using the particular structure of $\boldsymbol{E}^{(L)}(z)$ given in (4). Inserting (4) into (17) and rewriting the expression obtained 
using (10) yields

$$
\begin{aligned}
\boldsymbol{S}(z)= & \frac{1}{L} \sum_{\ell=0}^{2 L-1} \boldsymbol{P}_{\ell}\left(z^{-2 L}\right) \boldsymbol{P}_{\ell}\left(z^{2 L}\right)+(-1)^{s} \frac{1}{L} \\
& \times \sum_{\ell=0}^{L-1} z^{L-1-2 \ell} \boldsymbol{P}_{L-1-\ell}\left(z^{-2 L}\right) \boldsymbol{J}_{N} \boldsymbol{P}_{\ell}\left(z^{2 L}\right)-(-1)^{s} \frac{1}{L} \\
& \times \sum_{\ell=0}^{L-1} z^{L-1-2 \ell} \boldsymbol{P}_{2 L-1-\ell}\left(z^{-2 L}\right) \boldsymbol{J}_{N} \boldsymbol{P}_{L+\ell}\left(z^{2 L}\right)
\end{aligned}
$$

Because the matrices $\boldsymbol{P}_{\ell}\left(z^{2 L}\right)$ are diagonal, it turns out that $\boldsymbol{S}(z)$ has nonzero elements on its diagonal and anti-diagonal only. Let us write $\boldsymbol{S}(z)$ as

$$
\begin{aligned}
\boldsymbol{S}(z)=\operatorname{diag}[ & \left.S_{0,0}(z), \ldots, S_{N-1, N-1}(z)\right] \\
& +\operatorname{diag}\left[S_{0, N-1}^{\prime}(z), \ldots, S_{N-1,0}^{\prime}(z)\right] \boldsymbol{J}_{N} .
\end{aligned}
$$

From (7) and (19), we find that

$$
S_{k, k}(z)=\frac{1}{L} \sum_{\ell=0}^{2 L-1} P_{k+\ell N}\left(-z^{-2 L}\right) P_{k+\ell N}\left(-z^{2 L}\right)
$$

and we have (22), shown at the bottom of the page. To simplify the further discussion, we first look at the case where $N$ is even, and then, we outline the changes for odd $N$.

Even $N$ : Because of the special structure of $\boldsymbol{S}\left(e^{j \omega}\right)$, its eigenvectors have only two nonzero entries, and we can reduce the task to that of analyzing the eigenvalues of the $2 \times 2$ matrices

$$
\begin{gathered}
\boldsymbol{S}_{k}\left(e^{j \omega}\right)=\left[\begin{array}{cc}
S_{k, k}\left(e^{j \omega}\right) & S_{k, N-1-k}^{\prime}\left(e^{j \omega}\right) \\
S_{N-1-k, k}^{\prime}\left(e^{j \omega}\right) & S_{N-1-k, N-1-k}\left(e^{j \omega}\right)
\end{array}\right] \\
k=0,1, \ldots, \frac{N}{2}-1
\end{gathered}
$$

A more detailed explanation is given in Appendix A. From (21) and (22), one can infer that the terms $S_{k, k}\left(e^{j \omega}\right)$ are real-valued and that $S_{k, N-1-k}^{\prime}\left(e^{j \omega}\right)$ are symmetric, i.e., $S_{k, k}\left(e^{j \omega}\right)=S_{k, k}\left(e^{-j \omega}\right)$ and $S_{k, N-1-k}^{\prime}\left(e^{j \omega}\right)=S_{N-1-k, k}^{\prime}\left(e^{-j \omega}\right)$. Hence, the two eigenvalues $\lambda_{k}(\omega)$ and $\lambda_{\lceil N / 2\rceil+k}(\omega)$ of $S_{k}\left(e^{j \omega}\right)$ can be explicitly expressed as ${ }^{2}$ (24), shown at the bottom of the page. Note that (24) provides a straightforward method for determining the eigenvalues from the polyphase components of the prototype. The required terms $S_{k, k}\left(e^{j \omega}\right)$ and $S_{k, N-1-k}^{\prime}\left(e^{j \omega}\right)$ are simply the discrete-time Fourier transforms of sequences $s_{k, k}(n)$ and $s_{k, N-1-k}^{\prime}(n)$, which can be obtained as follows. Let

$$
\phi_{k}(n)= \begin{cases}(-1)^{n} p_{k}\left(\frac{n}{2 L}\right), & \text { if } \frac{n}{2 L} \in \mathbb{Z} \\ 0, & \text { otherwise. }\end{cases}
$$

Then

$$
s_{k, k}(n)=\frac{1}{L} \sum_{\ell=0}^{2 L-1} \phi_{k+\ell N}(-n) * \phi_{k+\ell N}(n)
$$

and we have (27), shown at the bottom of the next page. For FIR prototypes, an FFT algorithm and zero padding can be used in practice to obtain $S_{k, k}\left(e^{j \omega}\right)$ and $S_{k, N-1-k}^{\prime}\left(e^{j \omega}\right)$ from $s_{k, k}(n)$ and $s_{k, N-1-k}^{\prime}(n)$ in an efficient manner on a fine frequency grid. A discussion of the discretization error is given in Appendix B.

Odd $N$ : For odd $N$, the formulation (20) with $S_{k, k}(z)$ and $S_{k, N-1-k}^{\prime}(z)$ according to (21) and (22) is still valid. However, because the diagonal and anti-diagonal of $S(z)$ meet in the center, there is a row and column containing a single nonzero entry $S_{c}(z)=S_{\lfloor N / 2\rfloor,\lfloor N / 2\rfloor}(z)+S_{\lfloor N / 2\rfloor,\lfloor N / 2\rfloor}^{\prime}(z)$. Thus, the eigenvalues of $\boldsymbol{S}\left(e^{j \omega}\right)$ are given by (24) for $k=0,1, \ldots,\lfloor N / 2\rfloor-1$, and the extra value $\lambda_{\lfloor N / 2\rfloor}(\omega)=S_{c}\left(e^{j \omega}\right)$.

Relation to the Work in [12]: Frame bounds for oversampled cosine-modulated filterbanks were also considered in [12]. There, an alternative expression for the frame bounds of cosine-modulated filterbanks with integer oversampling was derived, which involves modulated versions of the prototype's polyphase components. It was shown that under certain symmetry conditions, which are satisfied by symmetric prototypes, the frame bounds can be obtained as suprema and infima of certain functions of frequency, which play the same role as the eigenvalues $\lambda_{\lceil N / 2\rceil+k}(\omega)$ in (24). However, (24) is not limited to symmetric prototypes and gives, therefore,

${ }^{2}$ The ceiling operation for the index has been introduced in view of the case where $N$ is odd.

$$
\begin{aligned}
S_{k, N-1-k}^{\prime}(z)= & \frac{(-1)^{s}}{L} \\
& \times \sum_{\ell=0}^{L-1} z^{L-1-2 \ell} P_{(L-1-\ell) N+k}\left(-z^{-2 L}\right) P_{\ell N+N-1-k}\left(-z^{2 L}\right)-\frac{(-1)^{s}}{L} \\
& \times \sum_{\ell=0}^{L-1} z^{L-1-2 \ell} P_{(2 L-1-\ell) N+k}\left(-z^{-2 L}\right) P_{(L+\ell) N+N-1-k}\left(-z^{2 L}\right) .
\end{aligned}
$$

$$
\begin{aligned}
\lambda_{k ;\lceil N / 2\rceil+k}(\omega)= & \frac{1}{2}\left(S_{k, k}\left(e^{j \omega}\right)+S_{N-1-k, N-1-k}\left(e^{j \omega}\right)\right) \\
& \pm \frac{1}{2} \sqrt{\left(S_{k, k}\left(e^{j \omega}\right)-S_{N-1-k, N-1-k}\left(e^{j \omega}\right)\right)^{2}+4\left|S_{k, N-1-k}^{\prime}\left(e^{j \omega}\right)\right|^{2}} .
\end{aligned}
$$


a more general formulation of the eigenvalues of the frame operator. For example, (24) also allows the frame analysis of low-delay filterbanks, which generally require nonsymmetric prototypes. When imposing symmetry of the form $P_{k}(z)= \pm z^{-L_{P_{k}}} P_{2 M-1-k}\left(z^{-1}\right)$, the anti-diagonal of $\boldsymbol{S}(z)$ vanishes, and (24) reduces to $\lambda_{k}(\omega)=S_{k, k}\left(e^{j \omega}\right)$, which is an alternative expression to the one given in [12].

2) Frame Bounds for the Synthesis Filterbank: Let

$$
\boldsymbol{T}(z)=\boldsymbol{R}^{(L)}(z) \tilde{\boldsymbol{R}}^{(L)}(z) .
$$

The frame bounds $A^{\prime}$ and $B^{\prime}$ are given by

$$
\begin{aligned}
& A^{\prime}=\underset{\omega \in[0,2 \pi), k=0,1, \ldots, N-1}{\operatorname{essinf}} \theta_{k}(\omega) \\
& B^{\prime}=\underset{\omega \in[0,2 \pi), k=0,1, \ldots, N-1}{\operatorname{ess} \sup _{k}(\omega)}
\end{aligned}
$$

where $\theta_{k}(\omega)$ are the eigenvalues of $\boldsymbol{T}\left(e^{j \omega}\right)$.

Carrying out the same derivation as for the analysis side yields

$$
\begin{aligned}
\boldsymbol{T}(z)=\operatorname{diag}[ & \left.T_{0,0}(z), \ldots, T_{N-1, N-1}(z)\right] \\
& -\operatorname{diag}\left[T_{0, N-1}^{\prime}(z), \ldots, T_{N-1,0}^{\prime}(z)\right] \boldsymbol{J}_{N}
\end{aligned}
$$

where the terms $T_{k, k}(z)$ and $T_{k, N-1-k}^{\prime}(z)$ are defined according to $S_{k, k}(z)$ and $S^{\prime}{ }_{k, N-1-k}(z)$, respectively, with $Q_{k}(z)$ instead of $P_{k}(z)$. Thus, $\boldsymbol{S}(z)$ and $\boldsymbol{T}(z)$ have the same structure but different signs for the anti-diagonal terms.

Frame Bounds for Equal Analysis and Synthesis Prototypes: In the following, we consider the use of the same prototype for analysis and synthesis, resulting in $T_{k, k}(z)=S_{k, k}(z)$ and $T^{\prime}{ }_{k, N-1-k}(z)=S^{\prime}{ }_{k, N-1-k}(z)$. If $N$ is even, we then have to analyze the submatrices

$$
\begin{aligned}
\boldsymbol{T}_{k}\left(e^{j \omega}\right)= & {\left[\begin{array}{cc}
S_{k, k}\left(e^{j \omega}\right) & -S_{k, N-1-k}^{\prime}\left(e^{j \omega}\right) \\
-S_{N-1-k, k}^{\prime}\left(e^{j \omega}\right) & S_{N-1-k, N-1-k}\left(e^{j \omega}\right)
\end{array}\right] } \\
& k=0,1, \ldots, \frac{N}{2}-1
\end{aligned}
$$

which have the same eigenvalues as $\boldsymbol{S}_{k}\left(e^{j \omega}\right)$ in (23). This means that

$$
A^{\prime}=A, \quad B^{\prime}=B
$$

for even $N$ and equal prototypes on the analysis and synthesis sides.

If $N$ is odd, we have to analyze the submatrices $\boldsymbol{T}_{k}\left(e^{j \omega}\right)$ for $k=0,1, \ldots,\lfloor N / 2\rfloor-1$ and a single term $T_{c}\left(e^{j \omega}\right)=$ $S_{\lfloor N / 2\rfloor,\lfloor N / 2\rfloor}\left(e^{j \omega}\right)-S_{\lfloor N / 2\rfloor,\lfloor N / 2\rfloor}^{\prime}\left(e^{j \omega}\right)$ in the center of $\boldsymbol{T}(z)$. Because $T_{c}\left(e^{j \omega}\right)$ will, in general, be different from $S_{c}\left(e^{j \omega}\right)$, it will cause an eigenvalue $\theta_{\lfloor N / 2\rfloor}(\omega)=T_{c}\left(e^{j \omega}\right) \neq \lambda_{\lfloor N / 2\rfloor}(\omega)$. Whether or not this leads to bounds $A^{\prime}$ and $B^{\prime}$ that are different from $A$ and $B$ depends on the prototype in use.

Relationship to Dual Frames: In [14], synthesis filterbanks of the form

$$
\hat{\boldsymbol{R}}(z)=[\tilde{\boldsymbol{E}}(z) \boldsymbol{E}(z)]^{-1} \tilde{\boldsymbol{E}}(z)
$$

were analyzed. There, it was shown that these particular synthesis filters are associated with the inverse frame operator of the analysis filterbank and that they lead to a dual frame with frame bounds $A^{\prime}=1 / B$ and $B^{\prime}=1 / A$. However, for the oversampled case, the synthesis filterbank is not unique, and there are infinitely many sets of synthesis filters that provide PR with the same delay $D$. In general, the filters in $\hat{R}(z)$ are not modulated versions of a single prototype and cannot be as efficiently implemented as modulated filterbanks. Exceptions are for critical sampling and for cases where the analysis prototype exhibits certain symmetries [12]. The synthesis filters considered in the present paper, on the other hand, are explicitly designed to be modulated versions of a prototype. Therefore, $\hat{\boldsymbol{R}}(z)$ and $\boldsymbol{R}(z)$ represent, in general, different PR synthesis filterbanks and lead to different frame bounds. In the critically sampled case, however, $\hat{\boldsymbol{R}}(z)$ and $\boldsymbol{R}(z)$ are equal up to a delay and yield the same frame bounds.

3) Frame Bounds for Critical Sampling: In the following, we derive a simplified method for computing the frame bounds in the critically sampled case with even $M$ and use of the same prototype on the analysis and synthesis sides. For this, we analyze the product $\lambda_{k}(\omega) \lambda_{N / 2+k}(\omega)$ with $L=1$ and, thus, with $N=M$. This yields

$$
\begin{array}{r}
\lambda_{k}(\omega) \lambda_{M / 2+k}(\omega)=S_{k, k}\left(e^{j \omega}\right) S_{M-1-k, M-1-k}\left(e^{j \omega}\right) \\
-\left|S_{k, M-1-k}^{\prime}\left(e^{j \omega}\right)\right|^{2} .
\end{array}
$$

Inserting (21) and (22) into (34) and evoking the PR condition (12) with $P(z)=Q(z)$ yields

$$
\lambda_{k}(\omega) \lambda_{M / 2+k}(\omega)=1 \forall \omega .
$$

This interesting relationship immediately implies that

$$
A B=1
$$

for critically sampled PR cosine-modulated filterbanks that are derived from a single prototype because for a fixed $k \leq M / 2$, the eigenvalue $\lambda_{k}(\omega)$ will take on its infimum at the same frequency where $\lambda_{(M / 2)+k}(\omega)$ has its supremum. Note that a similar condition has been mentioned in [16] for two-channel filterbanks.

$$
\begin{aligned}
s_{k, N-1-k}^{\prime}(n)= & \frac{(-1)^{s}}{L} \sum_{\ell=0}^{L-1} \delta(n+L-1-2 \ell) * \phi_{(L-1-\ell) N+k}(-n) * \phi_{\ell N+N-1-k}(n) \\
& -\frac{(-1)^{s}}{L} \sum_{\ell=0}^{L-1} \delta(n+L-1-2 \ell) * \phi_{(2 L-1-\ell) N+k}(-n) * \phi_{(L+\ell) N+N-1-k}(n) .
\end{aligned}
$$


Equation (35) is also the key to further simplification of the computation of the frame bounds. From (24) and (35), it follows that

$$
\lambda_{M / 2+k}(\omega)=A_{k}(\omega)+\sqrt{A_{k}^{2}(\omega)-1}
$$

with

$$
A_{k}(\omega)=\frac{1}{2}\left(S_{k, k}\left(e^{j \omega}\right)+S_{M-1-k, M-1-k}\left(e^{j \omega}\right)\right) .
$$

Inserting (21) into (38) with $z=e^{j \omega}$ under consideration of $P_{k}\left(-e^{-j 2 \omega}\right) P_{k}\left(-e^{j 2 \omega}\right)=\left|P_{k}\left(e^{j(2 \omega+\pi)}\right)\right|^{2}$ yields

$$
\begin{aligned}
A_{k}(\omega)= & \frac{1}{2}\left[\left|P_{k}\left(e^{j(2 \omega+\pi)}\right)\right|^{2}+\left|P_{M+k}\left(e^{j(2 \omega+\pi)}\right)\right|^{2}\right. \\
& +\left|P_{M-1-k}\left(e^{j(2 \omega+\pi)}\right)\right|^{2} \\
& \left.+\left|P_{2 M-1-k}\left(e^{j(2 \omega+\pi)}\right)\right|^{2}\right] .
\end{aligned}
$$

For further simplification, we define

$$
A_{k}^{\prime}(\omega)=A_{k}\left(\frac{\omega-\pi}{2}\right)
$$

which is the discrete-time Fourier transform of the sequence

$$
\begin{aligned}
a_{k}^{\prime}(n)= & \frac{1}{2}\left[p_{k}(n) * p_{k}(-n)+p_{M+k}(n) * p_{M+k}(-n)\right. \\
& +p_{M-1-k}(n) * p_{M-1-k}(-n) \\
& \left.+p_{2 M-1-k}(n) * p_{2 M-1-k}(-n)\right] .
\end{aligned}
$$

Again, the terms $A_{k}^{\prime}(\omega)$ can be efficiently computed from $a_{k}^{\prime}(n)$ on a fine frequency grid using an FFT algorithm and zero padding. An analysis of the discretization error of the FFT method is given in Appendix B. The frame bounds finally amount to

$$
B=\beta+\sqrt{\beta^{2}-1}, A=\frac{1}{B}=\beta-\sqrt{\beta^{2}-1}
$$

with

$$
\beta=\operatorname{ess}_{\omega \in[0, \pi)}^{\operatorname{ess} \sup _{k=0,1, \ldots, M / 2-1}} A_{k}^{\prime}(\omega) .
$$

\section{EXAMPLES}

In this section, we compare the performance of various lowdelay prototype designs for cosine-modulated filterbanks with $M=8$ channels and a delay of $D=15$ taps. In all examples, the same prototype was used for analysis and synthesis. Prototypes were designed to minimize the stopband energy

$$
\Phi=\int_{\omega_{s}}^{\pi}\left|P\left(e^{j \omega}\right)\right|^{2} d \omega
$$

under the constraint (12). To ensure that (12) is satisfied, the lifting structure of [17] was used. The stopband edge frequency for the prototypes was chosen as $\omega_{s}=\pi / M$.

Note that the objective function (44) is widely used in the design of both paraunitary and biorthogonal filterbanks. The assumption behind this is that the modulated filters in the filterbank should have good properties as long as $P(z)$ has good

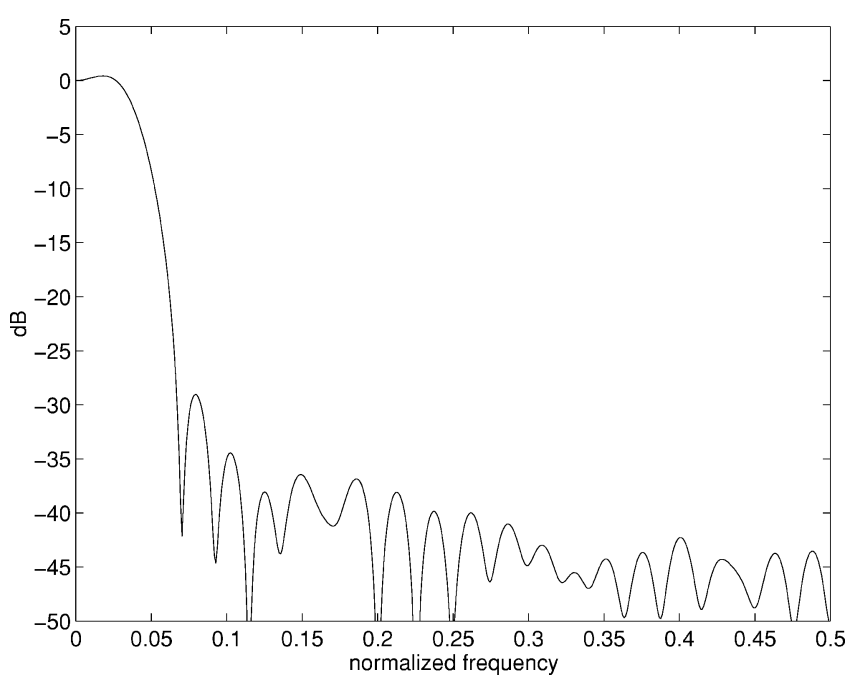

(a)

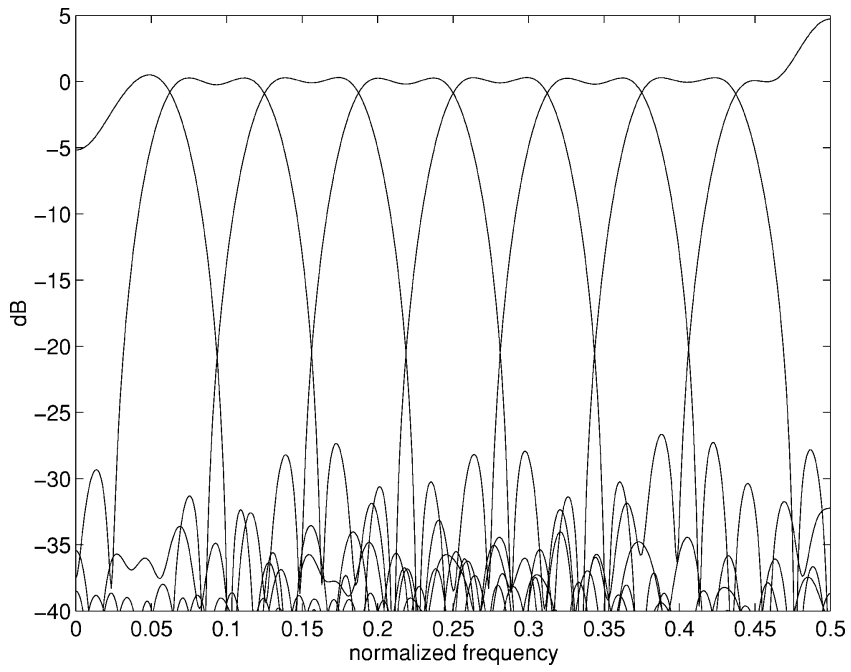

(b)

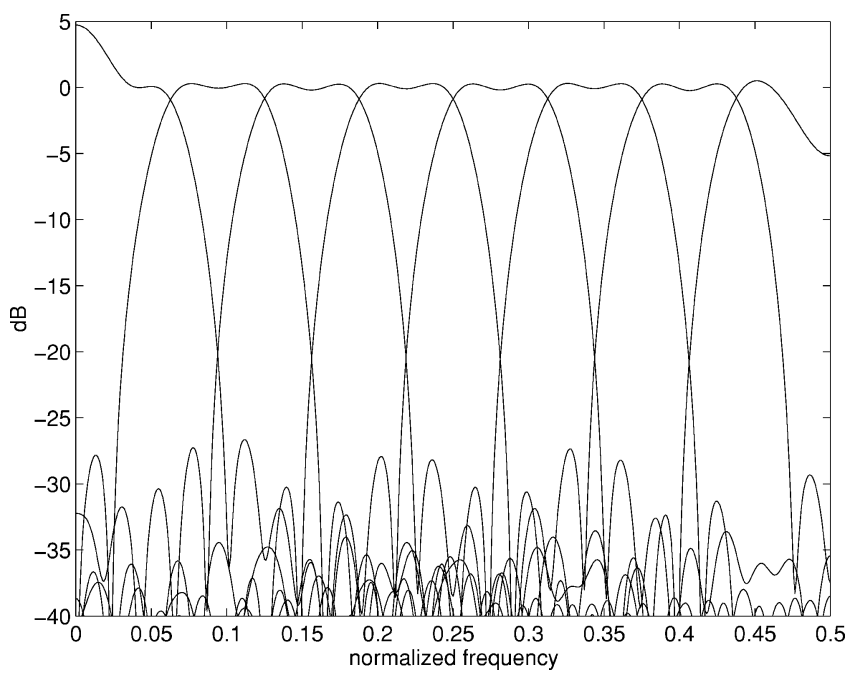

(c)

Fig. 2. Normalized frequency responses of eight-channel filterbank. (a) Prototype. (b) Analysis filters. (c) Synthesis filters. Parameters: $M=8$, $D=15, L_{p}=48$. Prototype designed to minimize (44).

properties. However, as the following examples will show, this is not necessarily true for biorthogonal filterbanks. 
TABLE I

Values $B, \Phi$, and $\Delta$ For Eight-ChanNel Filterbanks, Designed to Minimize (44) FOR CRitical SAMPLing

\begin{tabular}{c|c|c|c|c|c|c|c|c|c}
\hline$s / D$ & \multicolumn{3}{|c|}{$0 / 15$} & \multicolumn{3}{c|}{$1 / 31$} & \multicolumn{2}{c|}{$2 / 47$} & $3 / 63$ \\
\hline$L_{p}$ & 32 & 48 & 64 & 32 & 48 & 64 & 48 & 64 & 64 \\
\hline$B$ & 2.61 & 3.27 & 3.65 & 1.02 & 1.69 & 1.92 & 1.01 & 1.36 & 1.001 \\
$\Phi$ & 0.09 & 0.06 & 0.05 & 0.06 & 0.01 & 0.01 & 0.004 & 0.005 & 0.002 \\
$\Delta[\mathrm{dB}]$ & 7.58 & 9.91 & 11.02 & 0.01 & 4.47 & 5.57 & 0.01 & 2.72 & 0.01 \\
\hline
\end{tabular}

TABLE II

Values $B, \Phi$, And $\Delta$ For Eight-channel Filterbanks With $D=15$, Designed to Minimize (44) For Critical Sampling Under the CONDITION OF FIXED FRAME BOUNDS

\begin{tabular}{c|c|c|c|c|c|c|c|c|c|c|c|c}
\hline$L_{p}$ & \multicolumn{5}{|c|}{32} & \multicolumn{5}{c|}{48} & \multicolumn{4}{|c}{64} \\
\hline$B$ & 1.05 & 1.1 & 1.25 & 1.5 & 1.05 & 1.1 & 1.25 & 1.5 & 1.05 & 1.1 & 1.25 & 1.5 \\
$\Phi$ & 0.41 & 0.35 & 0.26 & 0.18 & 0.39 & 0.32 & 0.23 & 0.16 & 0.39 & 0.31 & 0.21 & 0.14 \\
$\Delta[\mathrm{dB}]$ & 0.28 & 0.69 & 1.79 & 3.2 & 0 & 0 & 1.55 & 3.32 & 0 & 0.32 & 1.62 & 3.18 \\
\hline
\end{tabular}

To design biorthogonal filterbanks with controlled frame bounds, we add the constraint

$$
B \leq B_{\max }
$$

during optimization, where $B_{\max }$ is an arbitrary, predefined upper bound for the actual frame bound $B$ with $1 \leq B_{\max }<\infty$. Due to (36), this implies that $A \geq 1 / B_{\max }$ for the case of critical sampling. For the constrained optimization, the Matlab routine fmincon has been used.

First, we look at designs using solely the objective function (44). The filter lengths $\left(L_{p}\right)$ are chosen as 32,48 , and 64, and critical sampling is considered. An example for the frequency response of a prototype filter is given in Fig. 2(a). One can see that this prototype has only a small ripple in the passband and a relatively high stopband attenuation. The frequency responses of the analysis filters derived from the prototype are depicted in Fig. 2(b). Interestingly, the near-ideal frequency response of the prototype does not translate into near-ideal modulated filters. The first and last filters $h_{0}(n)$ and $h_{M-1}(n)$ show a passband behavior that is significantly different from that of the prototype. The same holds for the corresponding synthesis filters $g_{0}(n)$ and $g_{M-1}(n)$; see Fig. 2(c). Experiments with various configurations (different $M, D, N$ ) showed that such a behavior is typical for cosine-modulated low-delay filterbanks with a prototype designed to minimize (44). Although the problems are concentrated on the extreme frequencies $\omega=0$ and $\omega=\pi$ for most lowpass prototypes, it is possible to design PR prototypes that show problems at other frequencies. In any case, the filters $h_{0}(n), g_{0}(n)$ and $h_{M-1}(n), g_{M-1}(n)$ are particularly critical. This becomes clear when expressing the real-valued cosine modulation with modulation frequencies $\omega_{k}=\pi(k+0.5) / M$ via Euler's formula as complex modulation with positive and negative frequencies $\pm \omega_{k}$. For $k=0$ and $k=M-1$, the frequency-shifted versions $P\left(e^{j\left(\omega \pm \omega_{k}\right)}\right)$ of the prototype show significant overlap. Depending on the relative phase, the overlap causes the increase and attenuation of the frequency response of $h_{0}(n), g_{0}(n), h_{M-1}(n)$, and $g_{M-1}(n)$.

To give a quantitative measure of the above-mentioned effect, we define the value $\Delta$ as the difference between the highest passband amplification in any one of the bands and the lowest local minimum in any of the passbands. If none of the passbands shows a local minimum, then $\Delta$ is set to zero. For the filterbank in Fig. 2, the value is $\Delta=9.91 \mathrm{~dB}$, which is a substantial variation within the passband frequency responses.

The frame bounds for the above-considered filterbank amount to $A=0.31$ and $B=3.27$, which means that the energy of the input or output signal of the filterbank may be different from the subband energy by a factor between 0.31 and 3.27, depending on the actual signal. Thus, although the filterbank constitutes a frame and provides PR, it is not well suited for schemes that rely on the assumption that the subband energy is close to the input/output signal energy. In addition, quantization noise introduced in the subbands may arrive at the output with an amplification of up to 3.27 (in terms of the noise power).

Table I gives an overview of the values $B, \Delta$, and $\Phi$ for eight-channel filterbanks with filter lengths of 32,48 , and 64 , and various delays. As the results show, for a fixed delay, the value of the objective function $\Phi$ decreases with increasing filter length, but the frame becomes more open, and the measure $\Delta$ for the passband variation increases. Thus, although increasing $N$ increases the stopband attenuation, it does not necessarily enhance the performance of the filterbank. The judgment certainly depends on the application. If, for example, the first and the last bands are not needed in an application and the filters $h_{1}(n), \ldots, h_{M-2}(n)$ have the desired frequency responses, then the behavior of $h_{0}(n)$ and $h_{M-1}(n)$ is irrelevant regarding the performance. If, however, the filterbank is used in a scheme that relies on the assumption of a near-unitary behavior, then a small $A$ and large $B$ pose problems. Table I also shows that with increasing delay, the performance is enhanced. For the delay of $D=L_{p}-1$, which is also achieved by paraunitary filterbanks with the same filter length, the above-mentioned problems disappear. However, increasing the delay over $L_{p}-1$ will cause the same problems as decreasing it.

We now consider the design of prototypes that minimize (44) under the condition of fixed frame bounds. Again, $M=8, D=$ 15 , and critical sampling are used. Table II gives an overview of 


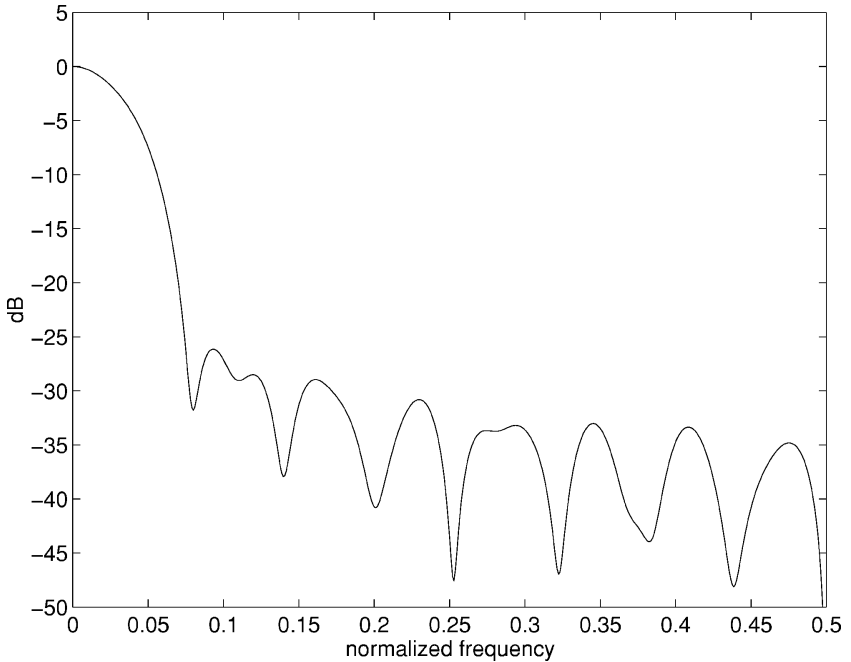

(a)

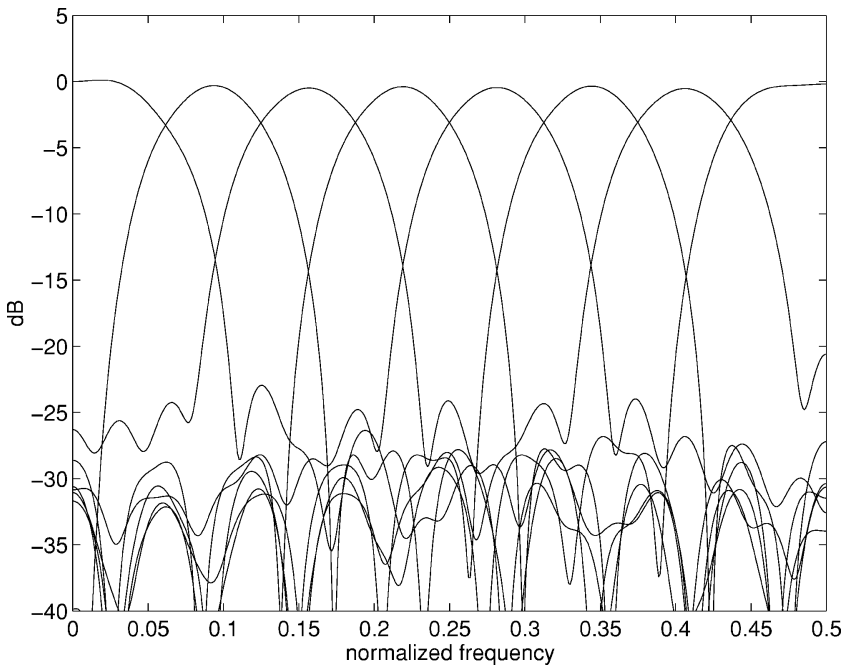

(b)

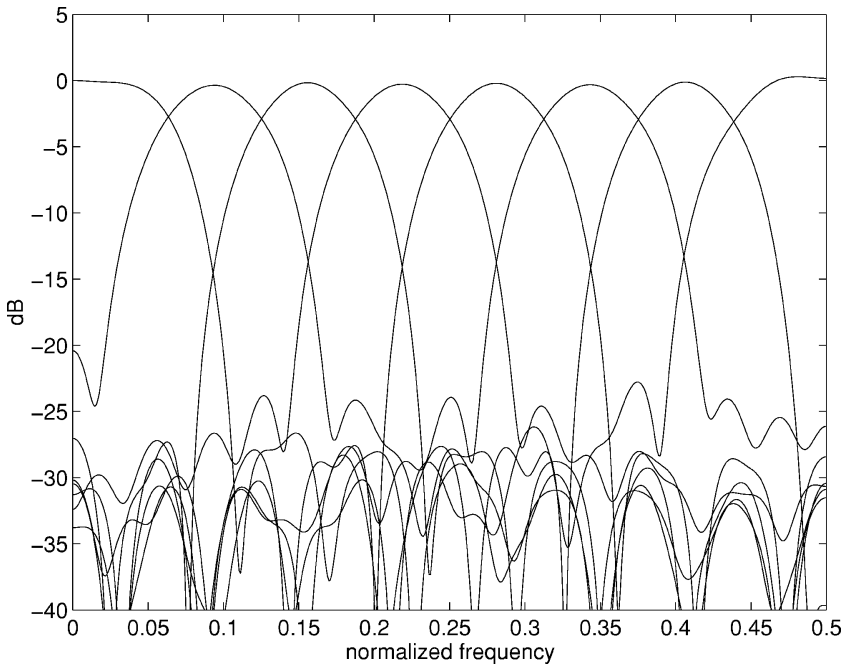

(c)

Fig. 3. Normalized frequency responses of eight-channel filterbank. (a) Prototype. (b) Analysis filters. (c) Synthesis filters. Parameters: $M=8$, $D=15, L_{p}=48$. Prototype designed to minimize (44) under the condition of $B=1.1$.

the obtained values $B, \Phi$, and $\Delta$. A comparison of the results in Table II shows that a tighter frame yields less passband vari-

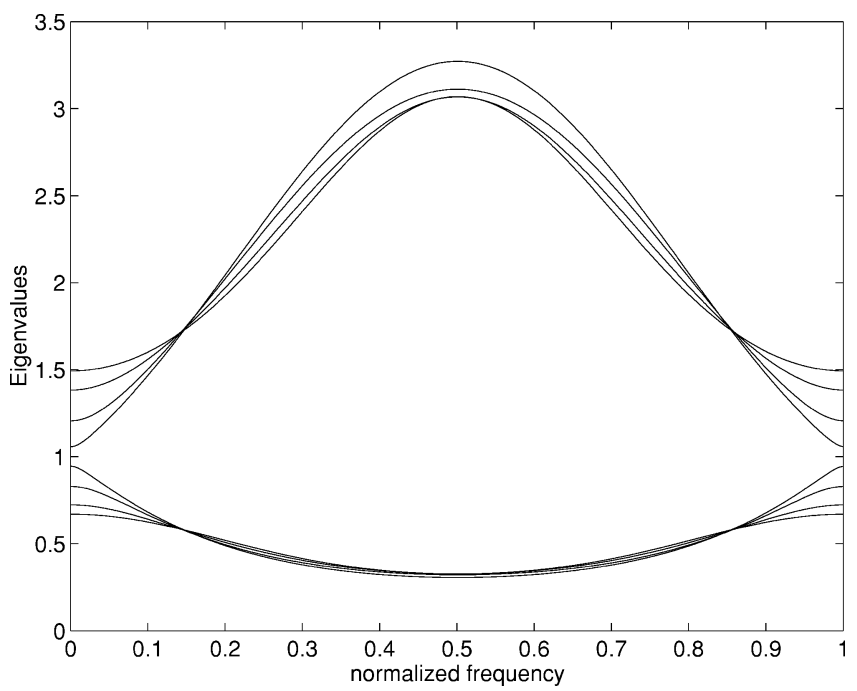

(a)

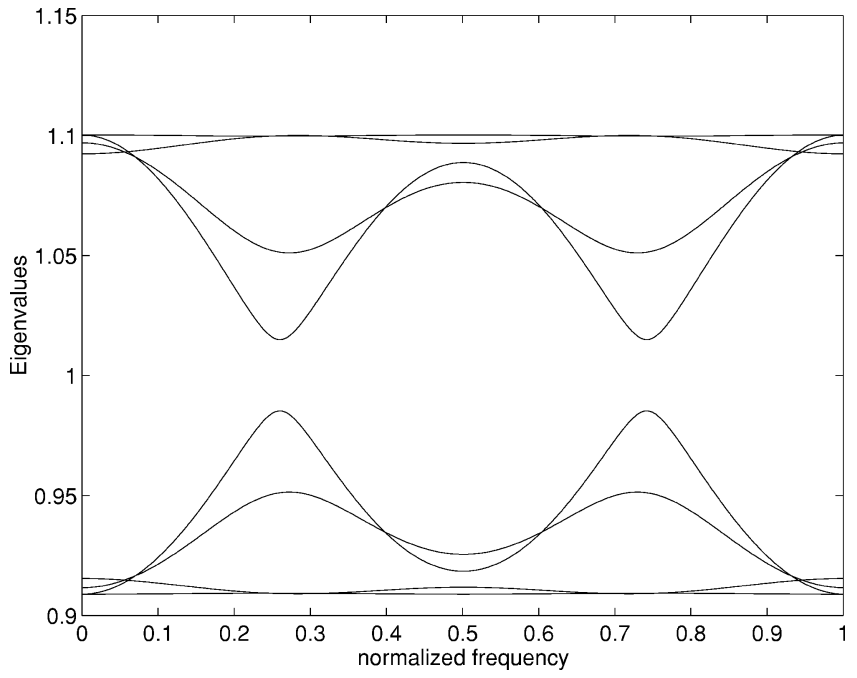

(b)

Fig. 4. Eigenvalues $\lambda_{k}(\omega)$ as a function of frequency. (a) Filterbank of Fig. 2. (b) Filterbank of Fig. 3 .

TABLE III

Frame Bounds For Filters Designed for CRITICAL SAMPLING AND USED WITH OVERSAMPLING FACTOR $L$. FILTER LENGTH $L_{p}=48$. INDICES INDICATE THE OVERSAMPLING FACTORS

\begin{tabular}{c|c|c|c|c}
\hline$B_{1}$ & 1.05 & 1.1 & 1.25 & 1.5 \\
$A_{1}$ & 0.95 & 0.91 & 0.8 & 0.67 \\
\hline$B_{2}$ & 1.04 & 1.09 & 1.2 & 1.48 \\
$A_{2}$ & 0.96 & 0.92 & 0.83 & 0.68 \\
\hline$B_{4}$ & 1.03 & 1.06 & 1.19 & 1.46 \\
$A_{4}$ & 0.98 & 0.95 & 0.85 & 0.69 \\
\hline$B_{8}$ & 1.02 & 1.06 & 1.17 & 1.46 \\
$A_{8}$ & 0.98 & 0.95 & 0.87 & 0.69 \\
\hline
\end{tabular}

ation $\Delta$ but more stopband energy $\Phi$ than a more open frame. Increasing the filter length yields a lower stopband energy at the same frame bounds. Thus, one clearly gains a benefit from increasing the filter length at fixed frame bounds.

To give an example for the design under the condition of fixed frame bounds, Fig. 3 shows the frequency responses of the prototype and the analysis and synthesis filters for the case where 
TABLE IV

Values $B, \Phi, \Delta$, And $A$ For Eight-Channel Filterbanks With $D=15$, Designed to Minimize (44) For Oversampling Factor Two, Under the CONDITION OF FIXED FRAME BOUNDS

\begin{tabular}{c|c|c|c|c|c|c|c|c|c|c|c|c}
\hline$L_{p}$ & \multicolumn{4}{|c|}{32} & \multicolumn{3}{c|}{48} & \multicolumn{4}{|c}{64} \\
\hline$B$ & 1.05 & 1.1 & 1.25 & 1.5 & 1.05 & 1.1 & 1.25 & 1.5 & 1.05 & 1.1 & 1.25 & 1.5 \\
$\Phi$ & 0.34 & 0.27 & 0.21 & 0.16 & 0.33 & 0.26 & 0.18 & 0.12 & 0.33 & 0.26 & 0.17 & 0.11 \\
$\Delta[\mathrm{dB}]$ & 0.41 & 0.79 & 1.9 & 3.5 & 0.41 & 0.79 & 1.9 & 3.5 & 0.41 & 0.76 & 1.9 & 3.6 \\
\hline
\end{tabular}

$L_{p}=48, D=15$, and $B=1.1$. As the graphs in Fig. 3(b) and (c) show, all filters $h_{k}(n)$ and $g_{k}(n)$ are almost ideally frequency-translated versions of the prototype. The value $B=1.1$ indicates a relatively tight frame so that the assumption of a near-unitary behavior can be justified in applications such as signal compression. In addition, the passband variation $\Delta$ is 0 $\mathrm{dB}$ in this case.

To illustrate the control of the frame bounds during the design procedure, Fig. 4 shows the eigenvalues $\lambda_{k}(\omega)$ as a function of frequency for the examples in Figs. 2 and 3. While the extrema in Fig. 4(a) occur at $\omega=\pi$, they appear in Fig. 4(b) at $0.57 \pi$. Thus, in general, the extrema do not necessarily occur at one of the special frequencies $\omega=0$ and $\omega=\pi$.

When using prototypes designed for critical sampling in oversampled filterbanks, the frames become tighter. This is demonstrated in Table III, which shows the frame bounds for the length-48 filters of Table II and different oversampling factors. Table III also shows the lower bound $A$ because $A B=1$ only holds for critical sampling. In all cases, it turned out that $A B>1$ if $L>1$.

Finally, prototypes for oversampling by a factor two have been designed. To exploit the additional degrees of freedom due to oversampling, the lifting structure of [17] has been amended by $2 L \times 2 L$ rotation matrices following each lifting stage and taking linear combinations of the filters contributing to $P_{k+\ell N}(z), \ell=0,1, \ldots, 2 L-1$ for a given $k$. Correspondingly, the inverse rotation matrices were used together with the dual lifting stages for the design of $P_{2 M-1-k-\ell N}(z)$, $\ell=0,1, \ldots, 2 L-1$, and the same $k$. The completeness of this parameterization in the sense that it describes all possible prototypes for oversampled, cosine-modulated PR filterbanks has not been investigated, but it is clear that it increases the design freedom compared with critical sampling. The obtained values $B, A, \Phi$, and $\Delta$ are listed in Table IV. The comparison with Table II shows that oversampling allows us to obtain significantly smaller stopband energies for fixed frame bounds. This is partly due to the increased design freedom in the oversampled case but also due to the fact that for a given prototype, the frame becomes tighter with increasing oversampling factor.

\section{CONCLUSIONS}

Explicit expressions for the eigenvalues of the frame operator of cosine-modulated filterbanks have been presented, which allow an efficient computation of frame bounds directly from the prototype's polyphase components. The solutions hold for all numbers of channels and all integer oversampling factors. The analysis of low-delay, biorthogonal filterbanks showed that prototypes solely designed to minimize the stopband energy may lead to wide open frames and thus to an undesirable numerical behavior. The numerical properties can be directly controlled by incorporating a frame analysis into the prototype design process. The results show that increasing the filter length allows the lowering of the stopband energy at a given system delay and given frame bounds.

\section{APPENDIX A \\ EIGENVALUES AND EIGENVECTORS OF $\boldsymbol{S}\left(e^{j \omega}\right)$}

We consider $2 \times 2$ matrices of the form

$$
S_{k}=\left[\begin{array}{ll}
a_{k} & b_{k} \\
b_{k}^{*} & c_{k}
\end{array}\right]
$$

with $a_{k}, c_{k} \in \mathbb{R}$ and $b_{k} \in \mathbb{C}$. The eigenvalues $\lambda_{k}^{(\mu)}, \mu=1,2$ of $S_{k}$ are given by

$$
\lambda_{k}^{(1 ; 2)}=\frac{1}{2}\left(a_{k}+c_{k}\right) \pm \frac{1}{2} \sqrt{\left(a_{k}-c_{k}\right)^{2}+4\left|b_{k}\right|^{2}} .
$$

The eigenvectors of $\boldsymbol{S}_{k}$, which are denoted as $\boldsymbol{x}_{k}^{(\mu)}:=$ $\left[x_{k}^{(\mu)}, y_{k}^{(\mu)}\right]^{T}, \mu=1,2$, satisfy $\boldsymbol{S}_{k} \boldsymbol{x}_{k}^{(\mu)}=\lambda_{k}^{(\mu)} \boldsymbol{x}_{k}^{(\mu)}$.

Now, we consider an $N \times N$ matrix $\boldsymbol{S}$ with even $N$ defined as

$$
\begin{aligned}
\boldsymbol{S}= & \operatorname{diag}\left[a_{0}, a_{1}, \ldots, a_{N / 2-1}, c_{N / 2-1}, c_{N / 2-2}, \ldots, c_{0}\right] \\
& +\operatorname{diag}\left[b_{0}, b_{1}, \ldots, b_{N / 2-1}, b_{N / 2-1}^{*}, b_{N / 2-2}^{*}, \ldots, b_{0}^{*}\right] \boldsymbol{J}_{N} .
\end{aligned}
$$

It is easily verified that the length- $N$ vectors

$$
\boldsymbol{y}_{k}^{(\mu)}=\left[\mathbf{0}_{k}, x_{k}^{(\mu)}, \mathbf{0}_{N-2 k-2}, y_{k}^{(\mu)}, \mathbf{0}_{k}\right]^{T}
$$

where $\mathbf{0}_{k}$ denotes a row vector of $k$ zeros, and satisfies

$$
\boldsymbol{S} \boldsymbol{y}_{k}^{(\mu)}=\lambda_{k}^{(\mu)} \boldsymbol{y}_{k}^{(\mu)} \text {. }
$$

Thus, $\boldsymbol{y}_{k}^{(\mu)}$ are the eigenvectors, and $\lambda_{k}^{(\mu)}$ are the eigenvalues of $S$. The comparison with Section III-B shows that for even $N$, the eigenvalues of $\boldsymbol{S}\left(e^{j \omega}\right)$ are indeed given by (24).

For odd $N$, we have to consider a matrix $\boldsymbol{S}$ of the form

$$
\begin{aligned}
S= & \operatorname{diag}\left[a_{0}, \ldots, a_{\lfloor N / 2\rfloor-1}, a_{\lfloor N / 2\rfloor}, c_{\lfloor N / 2\rfloor-1}, \ldots, c_{0}\right] \\
& +\operatorname{diag}\left[b_{0}, \ldots, b_{\lfloor N / 2\rfloor-1}, b_{\lfloor N / 2\rfloor}, b_{\lfloor N / 2\rfloor-1}^{*}, \ldots, b_{0}^{*}\right] \boldsymbol{J}_{N} .
\end{aligned}
$$

In this case, $N-1$ eigenvectors of $\boldsymbol{S}$ have the same structure as for even $N$, and the corresponding eigenvalues are the same as above. One eigenvector is given by $\boldsymbol{y}=\left[\mathbf{0}_{\lfloor N / 2\rfloor}, 1, \mathbf{0}_{\lfloor N / 2\rfloor}\right]^{T}$, and the corresponding eigenvalue is $a_{\lfloor N / 2\rfloor}+b_{\lfloor N / 2\rfloor}$. Altogether, this shows that the computation of the eigenvalues of $\boldsymbol{S}\left(e^{j \omega}\right)$ can be restricted to the analysis of $2 \times 2$ and $1 \times 1$ submatrices of $\boldsymbol{S}\left(e^{j \omega}\right)$. 


$$
\begin{aligned}
\Delta_{A_{k}^{\prime}, \mu}^{2} & :=\left|A_{k}^{\prime}\left(\frac{2 \pi(\mu+1)}{\tilde{L}_{k}}\right)-A_{k}^{\prime}\left(\frac{2 \pi \mu}{\tilde{L}_{k}}\right)\right|^{2} \\
& =\left|\sum_{n=0}^{L_{k}-1} a_{k}^{\prime}\left(n-\frac{\left(L_{k}-1\right)}{2}\right)\left[e^{-j 2 \pi(\mu+1) n / \tilde{L}_{k}}-e^{-j 2 \pi \mu n / \tilde{L}_{k}}\right]\right|^{2} .
\end{aligned}
$$

\section{APPENDIX B \\ DISCRETIZATION ERROR OF THE FFT METHOD}

We study the discretization error of the FFT method for critical sampling. This error can be seen as an upper limit for the oversampled case because oversampling typically leads to tighter frames and, thus, to less frequency deviation of the eigenvalues of the frame operator. In particular, we consider the squared difference of two adjacent frequency samples of $A_{k}^{\prime}(\omega)$, computed with the FFT algorithm from the sequence $a_{k}^{\prime}(n)$ given in (41). Let $L_{k}$ be the length of $a_{k}^{\prime}(n)$, and let $\tilde{L}_{k} \geq L_{k}$ be the FFT length after zero padding. The squared discretization step size between frequencies $\omega_{\mu}=2 \pi \mu / \tilde{L}_{k}$ and $\omega_{\mu+1}=2 \pi(\mu+1) / \tilde{L}_{k}$ can be written as the equation at the top of the page. Using the Cauchy-Schwartz inequality and the fact that $\left|e^{j \varphi}\right|=1$, we obtain

$$
\Delta_{A_{k}^{\prime}, \mu}^{2} \leq\left\|\boldsymbol{a}_{k}^{\prime}\right\|^{2} \cdot \varepsilon^{2}\left(L_{k}, \tilde{L}_{k}\right)
$$

with $\left\|\boldsymbol{a}_{k}^{\prime}\right\|^{2}=\sum_{n=0}^{L_{k}-1}\left|a_{k}^{\prime}\left(n-\left(L_{k}-1\right) / 2\right)\right|^{2}$ and $\varepsilon^{2}\left(L_{k}, \tilde{L}_{k}\right)=\sum_{n=0}^{L_{k}-1}\left|e^{-j 2 \pi n / \tilde{L}_{k}}-1\right|^{2}$. In order to ensure that $\Delta_{A_{k}^{\prime}, \mu}$ is smaller or equal to a maximum value $\Delta_{\max }$, we have to choose $\tilde{L}_{k}$ large enough to satisfy $\varepsilon\left(L_{k}, \tilde{L}_{k}\right) \leq \Delta_{\max } /\left\|\boldsymbol{a}_{k}^{\prime}\right\|$. To give an example, in the design of the prototype in Fig. 3, we have $L_{k}=3$ and $\left\|\boldsymbol{a}_{k}^{\prime}\right\|<2$. The above analysis shows that an FFT length of 1024 ensures that $\Delta_{A_{k}^{\prime}, \mu}<0.028$.

\section{REFERENCES}

[1] H. S. Malvar, "Extended lapped transforms: Fast algorithms and applications," IEEE Trans. Signal Processing, vol. 40, pp. 2703-2714, Nov. 1992.

[2] T. A. Ramstad and J. P. Tanem, "Cosine modulated analysis-synthesis filter bank with critical sampling and perfect reconstruction," Proc. IEEE Int. Conf. Acoust., Speech, Signal Process., pp. 1789-1792, May 1991.

[3] R. D. Koilpillai and P. P. Vaidyanathan, "Cosine-modulated FIR filter banks satisfying perfect reconstruction," IEEE Trans. Signal Processing, vol. 40, pp. 770-783, Apr. 1992.

[4] P. P. Vaidyanathan, Multirate Systems and Filter Banks. Englewood Cliffs, NJ: Prentice-Hall, 1993.

[5] K. Nayebi, T. P. Barnwell III, and M. J. T. Smith, "Low delay FIR filterbanks: Design and evaluation," IEEE Trans. Signal Processing, vol. 42 , pp. 24-31, Jan. 1994.
[6] G. Strang and T. Nguyen, Wavelets and Filter Banks. Wellesley, MA: Wellesley-Cambridge, 1996.

[7] G. D. T. Schuller and M. J. T. Smith, "A new framework for modulated perfect reconstruction filter banks," IEEE Trans. Signal Processing, vol. 44, no. 8, pp. 1941-1954, Aug. 1996.

[8] T. Q. Nguyen and P. N. Heller, "Biorthogonal cosine-modulated filter banks," Proc. IEEE Int. Conf. Acoust., Speech, Signal Process., vol. 3, pp. 1471-1474, May 1996.

[9] J. Kliewer and A. Mertins, "Oversampled cosine-modulated filter banks with arbitrary system delay," IEEE Trans. Signal Processing, vol. 46, pp. 941-955, Apr. 1998.

[10] P. N. Heller, T. Karp, and T. Q. Nguyen, "A general formulation for modulated filter banks," IEEE Trans. Signal Processing, vol. 47, pp. 986-1002, Apr. 1999.

[11] F. Argenti and E. Del Re, "Design of biorthogonal M-channel cosinemodulated FIR/IIR filter banks," IEEE Trans. Signal Processing, vol. 48, pp. 876-881, Mar. 2000.

[12] H. Bölcskei and F. Hlawatsch, "Oversampled cosine modulated filterbanks with perfect reconstruction," IEEE Trans. Circuits Syst. II, vol. 45, pp. 1057-1071, Aug. 1998.

[13] I. Daubechies, Ten Lectures on Wavelets. Philadelphia, PA: SIAM, 1992.

[14] H. Bölcskei, F. Hlawatsch, and H. G. Feichtinger, "Frame-theoretic analysis of oversampled filter banks," IEEE Trans. Signal Processing, vol. 46, pp. 3256-3268, Dec. 1998.

[15] G. Cvetković and M. Vetterli, "Oversampled filter banks," IEEE Trans. Signal Processing, vol. 46, pp. 1245-1255, May 1998.

[16] F. M. de Saint-Martin, P. Siohan, and A. Cohen, "Biorthogonal filterbanks and energy preservation property in image compression," IEEE Trans. Image Processing, vol. 8, pp. 168-178, Feb. 1999.

[17] T. Karp, A. Mertins, and G. Schuller, "Efficient biorthogonal cosinemodulated filter banks," Signal Processing, vol. 81, pp. 997-1016, May 2001.

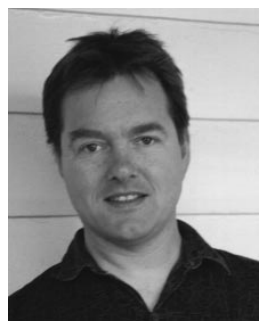

Alfred Mertins (M'95) received the Dipl.-Ing. degree (M.S.E.E.) from the University of Paderborn, Paderborn, Germany, in 1984 and the Dr.-Ing. degree (Ph.D.) in electrical engineering and the Dr.-Ing. hHabil. degree in telecommunications from the Hamburg University of Technology, Hamburg, Germany, in 1991 and 1994, respectively.

From 1986 to 1991, he was with the Hamburg University of Technology, from 1991 to 1995 with the Microelectronics Applications Center, Hamburg, from 1996 to 1997 with the University of Kiel, Kiel, Germany, and from 1997 to 1998 with the University of Western Australia, Perth. Since 1998 he has been with the School of Electrical, Computer and Telecommunications Engineering, University of Wollongong, Wollongong, Australia. His research interests include digital signal processing, wavelets and filterbanks, audio, image, and video processing, and digital communications. 